REVESCO. Revista de Estudios Cooperativos

ISSN: $1885-8031$

https://dx.doi.org/10.5209/REVE.69190

\title{
Análisis del impacto de las reformas financieras de 2014 en las sociedades cooperativas de ahorro y préstamo de México
}

\author{
Luis Miguel Cruz Lázaro ${ }^{1}$ y Felipe Abelardo Pérez $\operatorname{Sosa}^{2}(0$
}

Recibido: 2 de noviembre de 2019 / Aceptado: 24 de marzo de 2020 / Publicado: 4 de septiembre de 2020

Resumen. El objetivo de la investigación es determinar si las reformas de 2014 a la Ley para regular las actividades de las sociedades cooperativas de ahorro y préstamo, tuvieron un impacto en el crecimiento de las Sociedades Cooperativas de Ahorro y Préstamo de México que fomentaran la inclusión financiera. Para ello, se analizaron seis bases de datos de las Sociedades Cooperativas de Ahorro y Préstamo (SOCAPS) de México, dos de ellas relacionadas con el comportamiento de estas (número de sociedades y número de sucursales) y cuatro de ellas como variables proxis de la inclusión financiera (créditos vigentes y estos divididos en créditos comerciales, al consumo y a la vivienda). Estas seis bases de datos se analizaron empleando la prueba de estabilidad estructural de Chow y la prueba de análisis estructural con variables dicótomas, partiendo de la base de que la reforma financiera causó un cambio estructural en el año 2014. Complementariamente, también se analizó la tendencia en el comportamiento de las seis bases de datos usando la línea de tendencia de cada una de ellas. Los resultados indican que la reforma financiera de 2014 sí provocó un cambio estructural en cinco de las seis variables analizadas de las SOCAPS, también, que en los casos donde se presentó el cambio estructural, este fue tanto en la pendiente como en la ordenada al origen, es decir, afecta en la tendencia del comportamiento de las variables y en su número. Basándose en los resultados, se pudo concluir: a) que la reforma financiera de 2014 si causó un cambio estructural en las SOCAPS; b) la reforma financiera impactó de manera positiva en el crecimiento de la SOCAPS de México que causó un aumento en el número de sociedades y de sucursales; c) la reforma financiera incrementó la cantidad de créditos otorgados por las SOCAPS, lo cual impactó positivamente a la inclusión financiera; y, c) los objetivos por los cuales se instrumentó la reforma financiera de 2014 se cumplieron. Palabras clave: Análisis estructural; Sector financiero; Regulación financiera; Inclusión financiera; Ahorro y crédito popular; Crédito cooperativo.

Claves Econlit: C58; G21; G29; G51.

\section{[en] Analysis of the impact of the 2014 financial reforms on Mexican cooperative financial institutions}

Abstract. The aim of this paper is to determine if the 2014 reforms on the regulations of the Mexican Cooperative Financial Institutions, had an impact on the growth of such entities, wich facilitate financial inclusion. To this end, we analyzed six databases of the Cooperative Financial Institutions (SOCAPS in Spanish) from Mexico: two related to their behavior (number of companies and number of branches), and, four of them, as proxies for financial inclusion (loan accounts, divided into commercial, consumption and housing). These six databases were analyzed using the Chow test for structural stability, and the structural analysis with dummies, on the basis that the financial reform caused a structural change in 2014. In addition, we observed the trend in the behavior of the six databases from a graphical perspective. The results show the financial reform of 2014 actually caused a structural change in five of the six analyzed variables, as well, that, in the cases where the structural change occurs, this was both in the slope and in the ordinate, thus, this affected both the trend, and the number of variables. Based on this results, we concluded: a) the financial reform of 2014 actually caused a structural change in the SOCAPS; b) the financial reform had a positive impact on the growth of Mexican SOCAPS, that caused an increase in the number of companies and branches; c) the financial reform increased the amount of credits granted by SOCAPS, which positively impacts financial inclusion; and, c) the objectives of the 2014 financial reform were achieved.

Keywords: Structural analysis; Financial sector; Financial regulation; Financial inclusion; Popular savings and loans; Financial cooperatives.

Sumario. 1. Introducción. 2. Marco teórico. 3. Metodología. 4. Resultados. 5. Conclusiones. 6. Referencias bibliográficas.

1 Universidad Autónoma de Querétaro, México.

Dirección de correo electrónico: 1cruz227@alumnos.uaq.mx.

2 Tecnológico de Monterrey, México.

Dirección de correo electrónico: $\underline{\text { f.perez@ tec.mx. }}$ 
Cómo citar. Cruz Lázaro, L.M.; Pérez Sosa, F.A. (2020) Análisis del impacto de las reformas financieras de 2014 en las sociedades cooperativas de ahorro y préstamo de México. REVESCO. Revista de Estudios Cooperativos, vol. 135 , e69190. https://dx.doi.org/10.5209/reve.69190.

\section{Introducción}

En el año 2014 en México se realizó una importante reforma financiera, en la cual se modificaron 31 leyes y se creó una nueva. Esta reforma se llevó a cabo por múltiples fines, los cuales son: contribuir a ampliar el acceso al crédito; incrementar la oferta de recursos a la actividad productiva; también, regular las nuevas operaciones y servicios que las instituciones financieras estaban ofreciendo; implementar nuevas reglas para prevenir nuevos riesgos; e impactar de manera positiva en la inclusión financiera y en la educación financiera (Rodríguez y Dorantes, 2016).

Para alcanzar los múltiples fines de esta reforma, se crearon distintas estrategias. Por ejemplo, para alcanzar el objetivo de facilitar el acceso al crédito e incrementar la oferta crediticia, se pretendió lograrlo por medio de tres formas: por un lado, para ampliar la oferta de los créditos destinados a las empresas pequeñas y medianas, se decidió que se haría con la banca de desarrollo; por otro lado, con los créditos especializados se optó que se utilizaría a las Sociedades Financieras de Objeto Múltiple (SOFOMES); y, para aumentar la oferta y el acceso a los microcréditos, se eligió que se emplearían a las Sociedades Cooperativas de Ahorro y Préstamos (SOCAPS), las Sociedades Financieras Populares (SOFIPOS) y las Sociedades Financieras Comunitarias (SOFINCOS) (Rodríguez y Dorantes, 2016; LRASCAP, 2019; LACP, 2019).

Debido a lo anterior, se tuvieron que reformar diversas leyes, entre ellas, se modificó en varios de sus artículos a la Ley para Regular las Actividades de las Sociedades Cooperativas de Ahorro y Préstamo (LRASCAP) ya que como se mencionó, se quería usar a las SOCAPS como medio para influir de forma positiva en la inclusión financiera e incrementar el acceso al microcrédito y esta es la Ley que las regula (Rodríguez y Dorantes, 2016; LRASCAP, 2019; LACP, 2019).

Es importante señalar que la LRASCAP fue modificada en dos ocasiones durante el 2014, en este sentido, una de las reformas obedecía a los objetivos que perseguía la reforma financiera de 2014 , que como se destacó era ampliar e incrementar la oferta de los microcréditos. La segunda modificación, estaba encaminada a armonizar la ley de Fideicomiso que Administra el Fondo para el Fortalecimiento de Sociedades y Cooperativas de Ahorro y Préstamo y de Apoyo a sus Ahorradores con la LRASCAP, buscando proteger a los socios de las SOCAPS de distintos problemas que han surgido, como las malas practicas llevadas a cabo por los administradores de las mismas (Rodríguez y Dorantes, 2016; LRASCAP, 2019; LACP, 2019).

Se debe resaltar que la estrategia del gobierno estaba en línea con lo que los investigadores de las Sociedades Cooperativas han hallado. $\mathrm{Y}$ es que los estudiosos han evidenciado que las Sociedades Cooperativas de Ahorro y Préstamo fomentan la inclusión social y el desarrollo económico de los lugares donde operan. Entre los principales motivos que se han identificado por los cuales las sociedades cooperativas logran lo anterior, se encuentran: que atienden a los sectores de la sociedad que no tienen acceso a las instituciones financieras tradicionales como los Bancos; que su principal mercado se encuentra en los sectores de la sociedad que tienen menos recursos; y, porque tienen un impacto positivo en el desarrollo económico y social en las regiones donde tienen presencia (Cabrera, 2015; Rodríguez y Dorantes, 2016; Cobián et al., 2016). Por estas razones y su importancia, es que las sociedades cooperativas se han estudiado desde distintos puntos de vista en varias partes del mundo (Lara y Pérez, 2015; Cabrera, 2015; Cárdenas et al., 2015; Martínez, et al., 2016; Calvo y Paúl, 2010; Lara, 2018 a, 2018 b; Masís, et al., 2016).

Debido a lo anterior, es que en esta investigación se plantea como objetivo principal, determinar si las reformas de 2014 a la Ley para regular las actividades de las sociedades cooperativas de ahorro y préstamo, tuvieron un impacto en el crecimiento de las Sociedades Cooperativas de Ahorro y Préstamo de México que fomentaran la inclusión financiera. Para ello, esta investigación se estructuró de la siguiente manera: en la sección dos, se presenta un marco teórico donde se abordan los aspectos más relevantes para la investigación; en la sección tres, se muestra el marco metodológico realizado en el estudio; en el apartado cuatro, se presentan los resultados y se discuten; y, en la sección cinco, se muestran las conclusiones a las cuales se llegaron derivado de la investigación.

\section{Marco teórico}

\subsection{Sociedades Cooperativas de ahorro y préstamo de México}

Existen diferentes tipos de Sociedades Cooperativas en varios países del mundo como España, Costa Rica y Canadá. Al respecto, las de México tienden a ser parecidas a las que operan en Estados Unidos, Canadá y 
Europa. Lo anterior es debido a que las Sociedades Cooperativas de México, al momento de conformarse tomaron como ejemplo las que ya existían y operaban en Canadá y en las instituciones de crédito que hay en Estados Unidos (Figueroa y Magaña, 2018; Varga, 2015; Masís, et al. 2016; Lara, et al., 2017).

El antecedente histórico de las Sociedades Cooperativas de Ahorro y Préstamo (SOCAPS) de México son las cajas populares o también conocidas como cajas de ahorro. Estas surgen en México a principios de la década de los cincuenta y en un inicio estas fueron promovidas por la Iglesia Católica, teniendo como principal objetivo impactar de forma positiva en las condiciones de vida de la sociedad mexicana. Estas obtenían sus recursos por medio de sus usuarios, los cuales conseguían como principal beneficio recibir préstamos con atractivas tasas de interés (Figueroa y Magaña, 2018; Lara, et al., 2017).

Es así que las Sociedades cooperativas de México comenzaron sus operaciones en la Ciudad de México, para posteriormente expandirse a otras regiones del país. Lo anterior propició que para la década de los sesenta se creara la Confederación Mexicana de Cajas Populares, la cual tenía como fin unificar las distintas cajas populares que operaban en el país. Es importante señalar que en todo este periodo las sociedades cooperativas de México carecían de leyes o reglamentos, lo que provocó que estas se desarrollaran y se expandieran sin tener una figura jurídica legal que las sustentara, aunque, se debe indicar que esto no fue un impedimento para su propagación a varias zonas del país. Esta situación se mantuvo por varias décadas (Figueroa y Magaña, 2018; Lara, et al., 2017; Lara, 2010, 2012).

Posteriormente, ocurrió un evento que contribuyó a la formalización de las cajas populares. Este sucedió en 1991 cuando se reformó la Ley General de Organizaciones y Actividades Auxiliares del Crédito (LGOAAC), para crear la figura jurídica llamada Sociedades de Ahorro y Préstamo (SAP). Ante esto, las Sociedades Cooperativas comenzaron a adoptar esta figura para poder formalizarse y tener un sustento legal (Figueroa y Magaña, 2018; Lara, 2012).

Consecutivamente, en 1994 se creó la Ley General de Sociedades Cooperativas (LGSC), la cual, estableció que las cooperativas de ahorro y préstamos son una modalidad del consumo y que tienen preferentemente como actividad el ahorro y el préstamo. Ante la creación de esta Ley muchas sociedades que estaban en proceso de regulación para volverse SAP, adoptaron la figura jurídica de Cooperativas. Se debe destacar que para la realización de esta Ley, se llevaron a cabo 14 foros a nivel nacional y diversos estudios de las cooperativas de otros países (Figueroa y Magaña, 2018; Lara, 2012; Medina y Flores, 2015; Lara, et al., 2017).

Esa figura jurídica se mantuvo hasta 2001, que fue cuando se promulgó la Ley de Ahorro y Crédito Popular (LACP), que tenía entre sus objetivos establecer que la Comisión Nacional Bancaria y de Valores (CNBV) tuviera facultades para regular la actividad financiera de las diversas organizaciones populares que operaban en México, como: cajas de ahorro, cajas solidarias, microbancos, etc. Adicionalmente, se promovió que se contrataran organismos internacionales con el objeto de que certificaran a las Federaciones de Comités de Supervisión Auxiliar, los cuales son quienes estarían a cargo de la supervisión de las cooperativas de ahorro y préstamo (Figueroa y Magaña, 2018; Lara, 2012; Torres y Cano, 2019; Lara, et al., 2017).

Sin embargo, las cooperativas de México no adoptaron la LACP, por lo cual, para 2009 se promulgó la Ley para Regular las Actividades de las Sociedades Cooperativas de Ahorro y Préstamo (LRASCAP). Esta Ley reconoce que las Sociedades Cooperativas de Ahorro y Préstamo (SOCAPS) pertenecen al sector social de la economía. También, en el artículo primero de la misma Ley se establecen los cuatro objetivos que tienen las SOCAPS (Figueroa y Magaña, 2018; LRASCAP, 2019; Lara y Pérez, 2015; Rodríguez y Dorantes, 2016; Lara, 2018 a, 2018; Torres y Cano, 2019; Lara, et al., 2017). Entre ellos se encuentran: I) Regular, promover y facilitar la captación de fondos o recursos monetarios y su colocación mediante préstamos, créditos u otras operaciones (LRASCAP, 2019: 1); y, II) Regular, promover y facilitar las actividades y operaciones de estas últimas, su sano y equilibrio desarrollo (LRASCAP, 2019: 1).

\subsection{Tipos de Sociedades Cooperativas de ahorro y préstamo de México}

En la LRASCAP (2019) en su artículo 18, establece que existen cuatro diferentes niveles de Sociedades Cooperativas de Ahorro y Préstamo (SOCAPS). En el mismo artículo, se especifica que todas están sometidas a la supervisión y regulación de la Comisión Nacional Bancaria y de Valores (CNBV), de la Ley de la Comisión Nacional Bancaria y de Valores, del Comité de Supervisión Auxiliar, y, de la misma Ley. También que la CNBV es quien emitirá las disposiciones generales para los cuatro niveles de las SOCAPS. En estas disposiciones se establecerán las características de las operaciones que podrán realizar, los límites de estas y los requisitos para llevarlos a cabo, de acuerdo con el nivel de operaciones que le toca basándose en el artículo 19 de la misma Ley. 
También, que es la CNBV quien establecerá las disposiciones de carácter general para determinar los criterios que se utilizaran para designar cada uno de los cuatro niveles de operación de las SOCAPS. Para ello, la CNBV tendrá que considerar el monto de los activos marcados en el mismo artículo. Basándose en lo anterior, la clasificación es: las SOCAPS de nivel uno son aquellas que el monto de sus activos totales son inferiores a 10 millones de UDIS; las de nivel dos son las que sus activos totales se encuentran entre diez millones y cincuenta millones de UDIS; las de nivel tres son aquellas que sus activos tienen un valor de entre 50 y 250 millones de UDIS; por último, las de nivel cuatro son aquellas que el valor de sus activos están por encima de los 250 millones de UDIS (LRASCAP, 2019).

La clasificación anterior influye directamente en los servicios y operaciones que las SOCAPS pueden ofrecer. Es así que en el artículo 19 de la LRASCAP (2019), se establecen las operaciones que pueden realizar las SOCAPS de nivel uno, dos tres y cuatro. Las SOCAPS de nivel uno pueden realizar entre otras operaciones: a) Recibir depósitos de dinero a la vista, de ahorro, a plazo, retirables en días preestablecidos y retirables con previo aviso (LRASCAP, 2019: 12); b) Recibir préstamos y créditos de entidades financieras nacionales o extranjeras, organismos internacionales, instituciones integrantes de la Administración Pública Federal o Estatal, fideicomisos públicos, así como proveedores nacionales y extranjeros (LRASCAP, 2019: 12); y d) Expedir y operar tarjetas de débito y tarjetas recargables (LRASCAP, 2019: 12).

Las SOCAPS de nivel dos pueden llevar a cabo todo lo que hacen las de nivel uno, más: efectuar actividades de factoraje financiero con sus socios o por cuenta de ellos; ofrecer servicios de cajas de seguridad; prestar los servicios de abono y descuento en nóminas; y, comprar o vender monedas en ventanilla ya sea por su cuenta o de terceros. Las de nivel tres, pueden efectuar todo lo que hacen las de nivel uno y dos, y, también: realizar contratos de arrendamientos financieros con sus socios; y, ofrecer servicios de tesorería y caja (LRASCAP, 2019).

Por último, las SOCAPS de nivel cuatro están facultadas para realizar todas las operaciones que hacen las de nivel uno, dos y tres, y, además: pueden asumir obligaciones por terceros, basándose en créditos concedidos por medio del otorgamiento de aceptaciones, endoso o aval de títulos de crédito; pueden emitir tarjetas de crédito a sus socios a partir de contratos de apertura de crédito en cuenta corriente; y, dar descuentos de todo tipo, reembolsables en plazos congruentes con las operaciones pasivas que se llevan a cabo (LRASCAP, 2019).

\subsection{Reforma financiera de 2014 y las cooperativas de México}

Como se mencionó, en el año 2014 se realizó en México una importante reforma financiera, durante la cual se modificaron 31 leyes federales y se creó una nueva. Los objetivos oficiales de esta fueron: impactar de forma positiva en el acceso al crédito y aumentar la oferta de recursos para la actividad productiva; regular los nuevos servicios y operaciones que las instituciones financieras ofrecían; aplicar nuevas reglas para futuros riesgos; y, contribuir positivamente en la inclusión financiera y en la educación financiera (Rodríguez y Dorantes, 2016).

En específico, para poder ampliar y facilitar el acceso a las distintas modalidades de los créditos, se planteó hacerlo por medio de tres estrategias: por un lado, para aumentar y facilitar el acceso a los microcréditos se usarían a las SOCAPS, a las Sociedades Financieras Populares (SOFIPOS) y a las Sociedades Financieras Comunitarias (SOFINCOS); por otro lado, para los créditos especializados se planeaba usar a las Sociedades Financieras de Objeto Múltiple (SOFOMES); por último, para los créditos destinados a las medianas y grandes empresas, se pensó en usar a la Banca de Desarrollo. Lo anterior, implico que, para alcanzar este objetivo, el gobierno tuviera que reformar diversas leyes. Entre las que se encuentran: la Ley para Regular las Actividades de las Sociedades Cooperativas de Ahorro y Préstamo; la Ley de Ahorro y Crédito Popular; la Ley de Uniones de Crédito; la Ley de Instituciones de Crédito; la Ley orgánica de Sociedad Hipotecaria Federal; la Ley orgánica de Nacional Financiera; y otras (Rodríguez y Dorantes, 2016; LRASCAP, 2019; LACP, 2019).

Durante esta reforma financiera, la Ley para Regular las Actividades de las Sociedades Cooperativas de Ahorro y Préstamo (LRASCAP) fue modificada en dos ocasiones, la primera sucedió el 10 de enero y la segunda fue el 28 de abril del mismo año. La actualización que se realizó el 10 de enero tenía la finalidad de contribuir a que el sector de ahorro y crédito popular, en especial las Sociedades Financieras Populares, las Sociedades Financieras Comunitarias, y, las Sociedades Cooperativas de Ahorro y Préstamo, pudieran ampliar sus servicios y con ello generar un impacto positivo en la inclusión financiera. Para cumplir con este objetivo, a partir de esta reforma se les permitió poder contratar los servicios de terceros para poder extender sus servicios y se autorizó que estos pueden cobrar comisiones al realizar estas operaciones a su nombre o a nombre de las sociedades. La misma actualización creó los límites en los cuales pueden actuar los terceros o 
comisionistas en nombre de las sociedades, es importante señalar que en esta actualización se facultó a la CNBV para suspender parcial o total, y, temporal o definitivamente, a los prestadores de dichos servicios o comisionistas (LRASCAP, 2019; LACP, 2019; Rodríguez y Dorantes, 2016).

Por otro lado, la actualización que se llevó a cabo el 28 de abril de 2014 tenía como fin, armonizar la Ley que crea el Fideicomiso que Administra el Fondo para el Fortalecimiento de Sociedades y Cooperativas de Ahorro y Préstamo y de Apoyo a sus Ahorradores, llamada Ley del Fipago publicada en el año 2000 con la LRASCAP. Lo anterior, debido a que en esas fechas miles de socios habían sido defraudados por muchas sociedades que habían cerrado, derivado entre otros motivos por las malas prácticas de sus administradores, resaltando que, en el estado de Oaxaca, quince mil socios habían perdido sus escasos ahorros. Con esta ley se pretendía fortalecer a las sociedades apoyándolas y fortaleciéndolas por medio de los mecanismos establecidos en la ley del Fipago y también beneficiar a los socios de las organizaciones (LRASCAP, 2019; Rodríguez y Dorantes, 2016).

\section{Metodología}

La metodología desarrollada tiene como fin alcanzar el objetivo principal de la investigación que es determinar si las reformas de 2014 a la Ley para regular las actividades de las sociedades cooperativas de ahorro y préstamo, tuvieron un impacto en el crecimiento de las Sociedades Cooperativas de Ahorro y Préstamo de México que fomentaran la inclusión financiera. Por ello se propone una investigación de tipo cuantitativa (Ollivier y Thompson, 2017). Es así que con la finalidad de lograr el objetivo principal, primero se buscan las bases de datos de las Sociedades Cooperativas de Ahorro y Préstamo (SOCAPS) de México de variables que permitan determinar el impacto que tuvo la reforma financiera de 2014 en su comportamiento y en la inclusión financiera.

Para esta investigación se obtuvieron seis bases de datos extraídas de la Comisión Nacional Bancaria y de Valores (CNBV), las cuales se dividieron en dos partes. Por un lado, dos de ellas relacionadas con el comportamiento de las SOCAPS de México y que son: número de sociedades y número de sucursales. Es relevante indicar, que estas dos bases de datos se conformaron de los reportes emitidos por la CNBV y que estos se publican solo los meses de marzo, junio, septiembre y diciembre de cada año a partir de 2010. En este sentido las dos bases de datos tienen 4 meses por año y comienzan desde septiembre de 2009, por lo cual, en esta investigación ambas bases de datos contienen 37 observaciones y abarcan del periodo de septiembre de 2009 a marzo de 2019.

Por el otro lado, se encuentran cuatro bases de datos de los créditos otorgados por las SOCAPS de México, que son las variables proxis de la inclusión financiera. Una de ellas es la base de datos de la cartera de crédito vigente, $\mathrm{y}$, las otras tres corresponden a esta dividida en: créditos comerciales, créditos al consumo y créditos a la vivienda. Es importante señalar que en las cuatro bases de datos de los créditos, la información se encontraba en unidades monetarias (nominales), por ello, se deflactaron utilizando el Índice Nacional de Precios al Consumidor (INPC) de México usando como año base, el año 2018. Esto con el fin de que el análisis fuera más riguroso y se trabajara con unidades reales (la información del INPC se consiguió en el Instituto Nacional de Estadística y Geografía). También, se debe añadir que la periodicidad de las cuatro bases de datos es mensual, por lo cual, cada una tiene 113 observaciones y todas abarcan del periodo de enero de 2010 a mayo de 2019. Las seis bases de datos son globales, es decir, es de todas las SOCAPS que hay en México y a todas se les calculó la línea de tendencia empleando el software EXCEL.

\subsection{Aplicación de la prueba de Chow}

Para analizar si la reforma financiera de 2014 provocó un cambio estructural en el comportamiento de las SOCAPS de México y que afectara en la inclusión financiera, se optó por aplicar la prueba de Chow. Para ello se siguió la metodología mostrada en Gujarati y Porter (2010). De acuerdo con los autores, el primer paso es detectar si hubo o se presentó un evento que pudiera provocar que el comportamiento de las variables no permaneciera constante a lo largo de un tiempo, en este caso, este suceso es la reforma financiera de 2014. El segundo paso es realizar tres regresiones lineales, una de ellas es la regresión completa de toda la serie sin interrupción; $y$, las otras dos son las regresiones separadas por el evento que causó el cambio estructural, es decir, una antes del cambio estructural y la otra posterior al suceso que provocó el cambio estructural. Se debe indicar que se consideró al año 2014 como la fecha en que sucedió el evento que causó el cambio estructural. En este sentido, en la base de datos que corresponde al número de sociedades y al número de sucursales, se partió del supuesto de que el cambio estructural empezó en junio de 2014; por otro 
lado, en las bases de datos de los créditos, se estableció que el cambio estructural comenzó en febrero de 2014. Las tres regresiones lineales tienen la forma de la Ecuación 1.

$$
Y_{t}=\alpha_{1}+\alpha_{2} X_{t}+\alpha_{3} X_{1}+\alpha_{4} X_{2}+u_{1}
$$

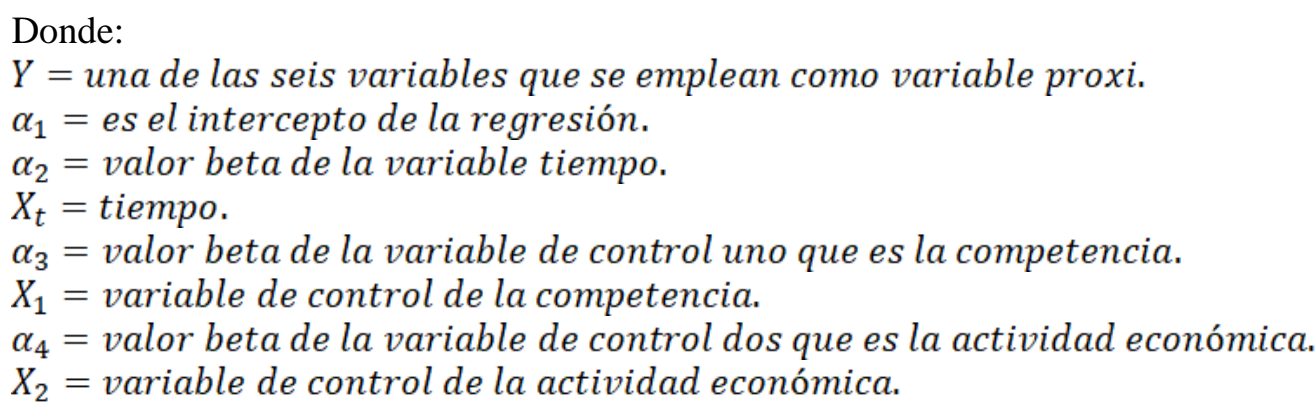

Se debe añadir que en la Ecuación 1 se aprecian las variables $X_{1}$ y $X_{2}$, estas se añadieron para que al momento de correr el modelo econométrico, estas se mantuvieran constante (es decir, se toma en consideración estas variables), por lo cual, los resultados fueran más representativos. Debido a lo anterior, se quiso tomar en consideración la competencia que tienen las SOCAPS y por ello se añadieron los créditos que emiten las instituciones bancarias de México. En este sentido la variable $X_{1}$ representa a la competencia, para lo cual se eligió a los créditos bancarios al consumo emitidos por las instituciones bancarias de México. La base de datos de esta variable fue extraída de Banco de México (BANXICO).

Respecto a la variable $X_{2}$ que es la que medirá la influencia que tiene la actividad económica en las SOCAPS, se optó por incorporar al Índice Global de la Actividad Económica (IGAE) ya que este índice mide la actividad económica y se reporta de manera mensual, en cambio, el Producto Interno Bruto (PIB) de México se reporta de forma trimestral lo cual no es conveniente para la investigación. La base de datos del IGAE fue extraída del Instituto Nacional de Estadística y Geografía (INEGI). Tanto los créditos bancarios como el IGAE fueron deflactados usando el INPC extraído del INEGI y la periodicidad es la misma que las de las seis bases de datos (es decir, se utilizan los mismos periodos).

Es importante señalar que por cada una de las seis bases de datos, se realizaron las tres regresiones lineales de acuerdo con el procedimiento descrito y que tienen la forma de la Ecuación 1. Una vez realizadas las regresiones lineales, se estimaron las Sumas de Cuadrados Residuales (SCR) de cada una de las tres regresiones. Las SCR de las dos regresiones que están separadas, se suman para crear la SCR no restringida $\left(S C R_{N R}\right)$ como se muestra en la Ecuación 2.

$S C R_{N R}=S C R_{1}+S C R_{2}$

Donde:

$S C R_{1}=$ la SCR antes del evento que causó el cambio estructural.

$S C R_{1}=$ la SCR después del evento que causó el cambio estructural.

Los datos obtenidos se sustituyen en la Ecuación 3 con el fin de obtener el valor $F$ calculado.

$F=\frac{\left(S C R_{R}+S C R_{N R}\right) / K}{\left(S C R_{N R}\right) /(n 1+n 2-(2 k)}$

Donde:

$k=$ número de variables de la regresión.

$n 1=$ número de observaciones antes del cambio estructural.

$n 2=$ número de observaciones después del cambio estructural.

Una vez estimada la $F$ calculada, se procede a buscar el valor $F$ crítico en las Tablas de la distribución $F$, está es con $K$ en el numerador (donde $K$ es el número de variables de la regresión, que en las seis regresiones es dos); y, $n 1+n 2-(2 k)$ en el denominador. Además de lo anterior, la $F$ crítica que se buscó en las tablas fue al 0.5 por ciento. Una vez estimadas la $F$ crítica y la $F$ calculada de las seis regresiones, se procede a compararlas por medio del criterio de decisión mostrada en la Ecuación 4. 
Rechazar la hipótesis de estabilidad paramétrica si: $F_{\text {crítica }}<F_{\text {calculada }}$

En la Ecuación 4 se presenta el criterio de decisión de la prueba de Chow, en donde se observa que si la $F$ calculada es mayor a la $F$ crítica, se rechaza la hipótesis de estabilidad estructural, es decir, sí hay un cambio estructural. Este criterio se aplica a las seis bases de datos para determinar si las reformas financieras de 2014 si causaron un cambio estructural o no.

\subsection{Análisis de cambio estructural con variables dicótomas}

Para sustentar y ampliar los resultados obtenidos de aplicar la prueba de Chow, se procedió a emplear una prueba de análisis estructural con variables dicótomas, ya que esta nos indicara si hubo un cambio estructural o no y si el cambio fue en la pendiente, en la ordenada al origen o en ambas. El procedimiento utilizado es el mostrado por Gujarati y Porter (2010), y se empleó en las seis bases de datos al igual que en la sección anterior. En este sentido de acuerdo con los autores se debe realizar una regresión múltiple que incluirá una variable dicótoma, por ello, en esta investigación se aplica una regresión múltiple como la mostrada en Ecuación 5.

$$
Y_{t}=\alpha_{1}+\alpha_{2} D_{t}+B_{1} T+B_{2}\left(D_{t} T\right)+B_{3} X_{1}+B_{4} X_{2}+u_{1}
$$

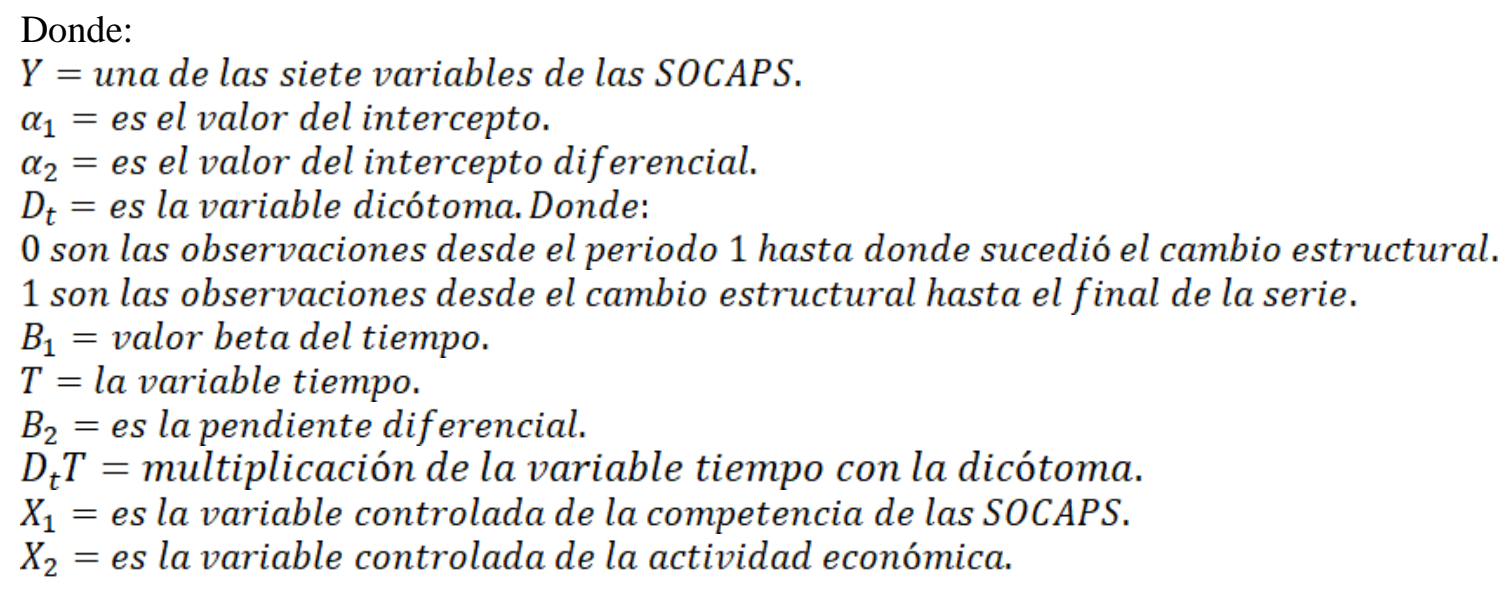

Se debe señalar que en la base de datos que corresponde al número de sociedades y al número de sucursales, se partió del supuesto de que el cambio estructural empezó en junio de 2014, es decir, antes de junio de 2014 la variable dicótoma tiene valor de cero y a partir de junio de 2014 es uno. Por otro lado, en las bases de datos de los créditos, se estableció que el cambio estructural comenzó en febrero de 2014, lo que indica que antes de febrero de 2014 la variable dicótoma tiene valor de cero y a partir de febrero de 2014 tiene el valor de uno.

Asimismo, al igual que en la prueba de Chow se añadieron dos variables de control con el fin de tomarlas en consideración y para que el análisis sea más completo. Para que los dos análisis se pudieran comparar y por cuestiones de la periodicidad de las bases de datos, se eligieron las mismas que en la prueba de Chow, las cuales son los créditos bancarios al consumo emitidos por las instituciones bancarias en México (información extraída de BANXICO) y el Índice Global de la Actividad Económica (IGAE) (los datos se consiguieron en el INEGI). Al igual que en la anterior prueba de análisis estructural, ambas bases de datos se deflactaron usando el INPC de México (la fuente de datos del INPC es INEGI) y la periodicidad de estas es la misma que las de las seis bases de datos analizadas (dicho de otra manera, estas concuerdan con cada una de las seis bases de datos analizadas).

Realizando la regresión múltiple con variables dicótomas de acuerdo con la Ecuación 5, se procede a analizar los valores $R^{2}$ obtenidos de cada una de ellas, con el fin de determinar si las regresiones son estadísticamente significativas o no, para lo cual, se toma como referencia que sean mayores al .8 que equivale al $80 \%$. Una vez identificado si el modelo es significativo, se procede a analizar los valores $p$ del intercepto diferencial $\left(\alpha_{2}\right)$ y de la pendiente diferencial $\left(B_{2}\right)$, los cuales deben ser menores a 0.05 para que sean estadísticamente significativos. Lo anterior es debido a que si el valor $p$ de ambas o de una sola es menor a 0.05 , indican que si hay un cambio estructural, pero, también señalan en donde fue el cambio estructural. En este sentido, si el valor $p$ del intercepto diferencial $\left(\alpha_{2}\right)$ es menor a 0.05 , entonces, el cambio estructural fue en el intercepto de la ordenada al origen; en cambio, si el valor $p$ de la pendiente diferencial 
$\left(B_{2}\right)$ es menor a 0.05 , el cambio es en la pendiente; por último, si ambos son menores a 0.05 , el cambio estructural fue tanto en la ordenada al origen como en el valor de la pendiente.

Este análisis se aplicó a las seis bases de datos, debido a que los resultados obtenidos de las seis pruebas de análisis estructural utilizando variables dicótomas, sustentan y complementa a los obtenidos por medio de la prueba de Chow. Los dos análisis se realizaron para poder comprobar si las reformas financieras si afectaron y causaron un cambio estructural en las SOCAPS de México. Por último, se procedió a analizar los resultados obtenidos de ambas pruebas.

\section{Resultados}

De acuerdo con la metodología mostrada en la sección anterior, primero se mostrarán las seis bases de datos obtenidas. En la Figura 1, se muestran las dos bases de datos que describen el comportamiento de las Sociedades Cooperativas de Ahorro y Préstamo (SOCAPS) de México y que corresponden al número de sociedades y al número de sucursales. Estas abarcan del periodo de septiembre de 2009 a marzo de 2019, en la misma figura se muestra la línea de tendencia de las dos bases de datos.

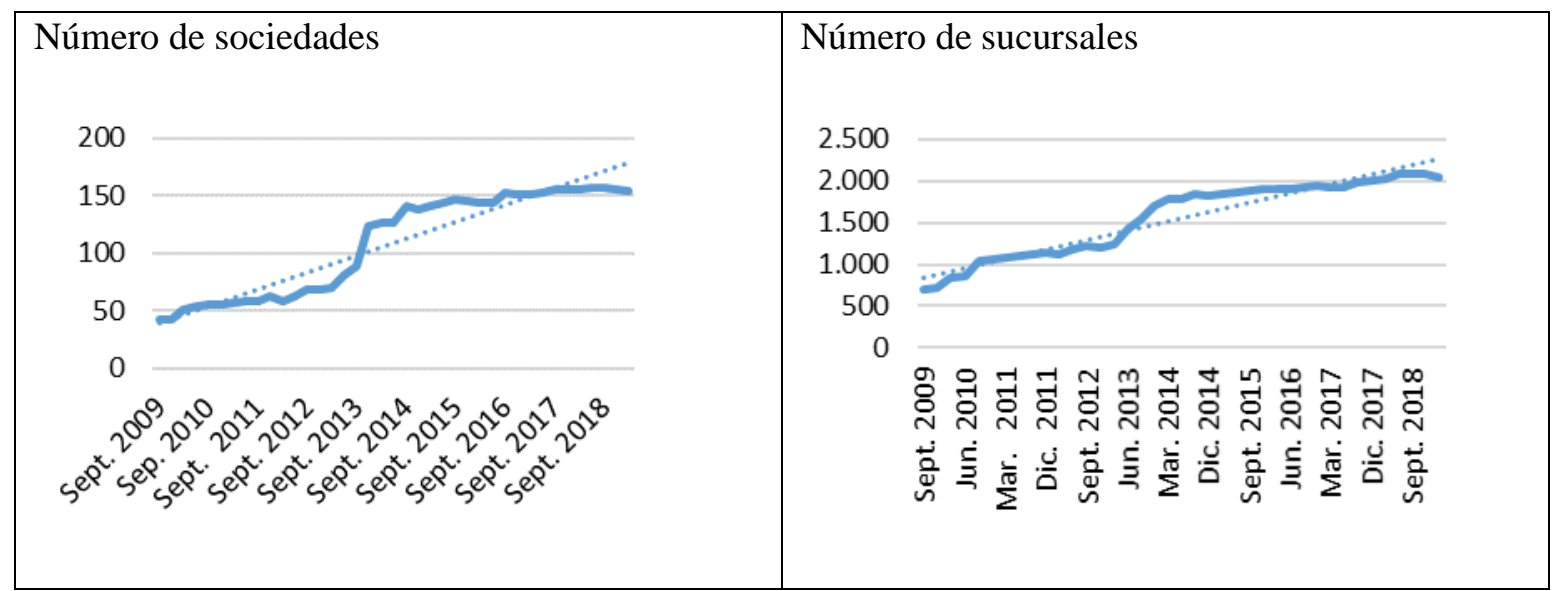

Figura. 1. Gráficos del comportamiento de las SOCAPS de México de 2009 a 2019. Fuente: Elaboración propia con base en CNBV.

De acuerdo con lo observado en la Figura 1, se aprecia que las SOCAPS de México han crecido y esto se sustenta por las líneas de tendencias de ambas bases de datos, las cuales tienen una pendiente positiva. Ahora se presentan en la Figura 2 las cuatro bases de datos correspondientes a las variables proxis de la inclusión financiera. Estas son la cartera de crédito vigente de las SOCAPS de México y está dividida en créditos comerciales, créditos al consumo y créditos a la vivienda. El periodo de las cuatro bases de datos es de enero de 2010 a mayo de 2019. En la misma Figura 2 se presenta la línea de tendencia de las cuatro bases de datos. 


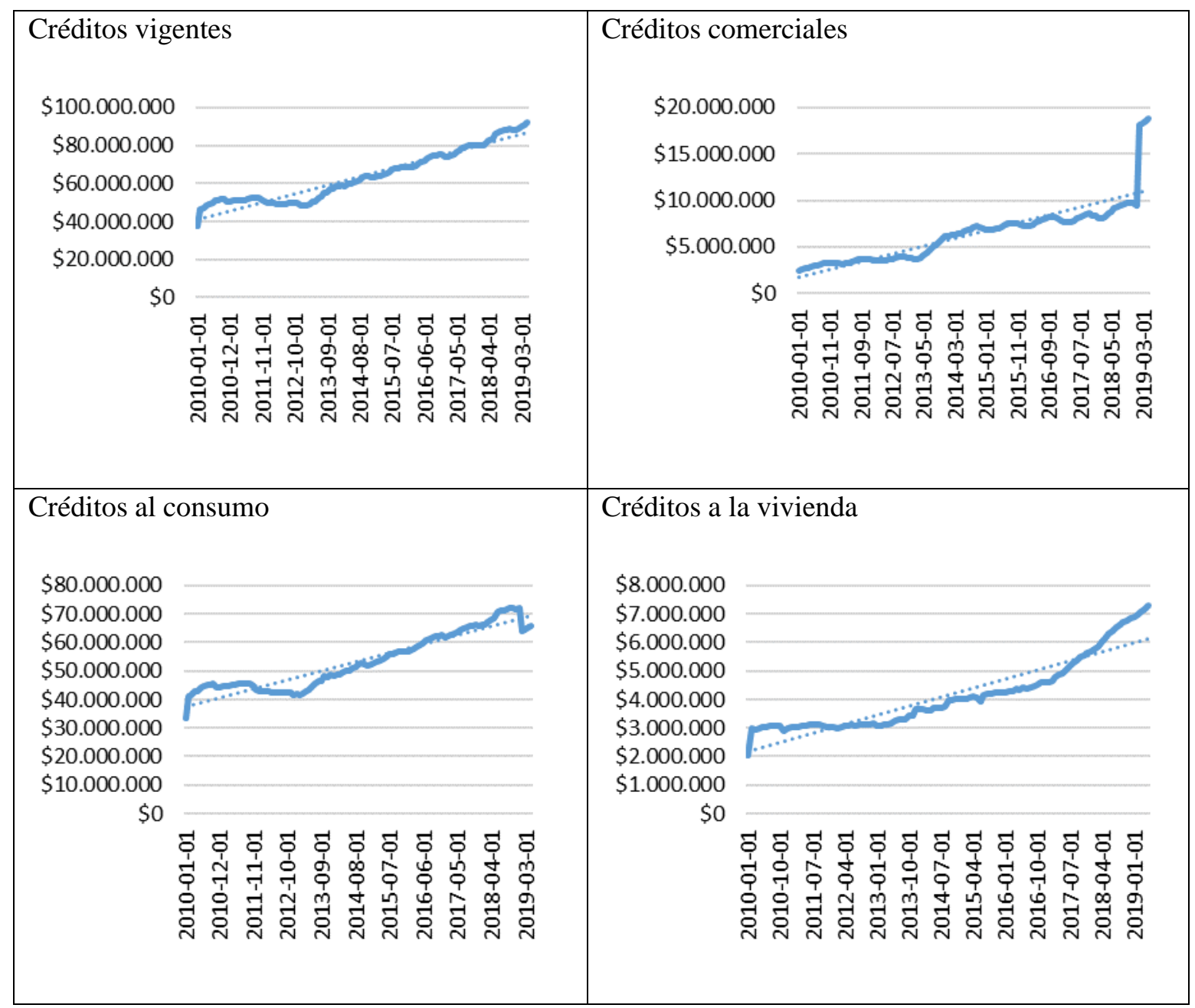

Figura. 2. Gráficos de la evolución de la cartera de crédito vigente de las SOCAPS de México y está dividida en créditos comerciales, créditos al consumo y créditos a la vivienda (miles de pesos). Fuente: Elaboración propia con base en CNBV.

En la Figura 2, se aprecia que los créditos emitidos por las SOCAPS de México han crecido. Lo anterior además de observarse, se basa en la línea de tendencia de las cuatro bases de datos, las cuales, tienen pendiente positiva lo que señala han tenido una tendencia creciente.

\subsection{Prueba de Chow}

Basándose en la metodología expuesta en la sección anterior, se procedió a aplicar la prueba de estabilidad estructural, es decir, la prueba de Chow para determinar si existe o no, un cambio estructural en las seis bases de datos mostradas previamente. Lo anterior con el fin de alcanzar el objetivo planteado en la investigación, que es determinar si las reformas de 2014 a la Ley para regular las actividades de las sociedades cooperativas de ahorro y préstamo, tuvieron un impacto en el crecimiento de las Sociedades Cooperativas de Ahorro y Préstamo de México. En la Tabla 1, se muestran los resultados del aplicar la prueba de Chow a las bases de datos del número de sucursales y de sociedades registradas de las SOCAPS de septiembre de 2009 a marzo de 2019 para analizar si hubo un cambio estructural por las reformas financieras de 2014 (en estas bases de datos el cambio estructural comenzó en junio de 2014). 
Tabla. 1. Resultados de la prueba de Chow (F) aplicado al número de sociedades y sucursales de las SOCAPS de México.

\begin{tabular}{llcc}
\hline Variable & Valor F calculado & Valor F crítico & Se rechaza la hipótesis de estabilidad estructural \\
\hline Número de sociedades & 62.2040099 & 3.284917651 & Si se rechaza \\
Número de sucursales & 73.56307211 & & \\
\hline
\end{tabular}

Fuente: Elaboración propia.

De acuerdo con los resultados mostrados en la Tabla 1 y que fueron obtenidos al aplicar la prueba estabilidad estructural, es decir, la prueba de Chow, a las bases de datos del número de sociedades y sucursales de las SOCAPS, se establece que si hubo un cambio estructural en ambas bases de datos de las SOCAPS de México en 2014. Debido a que los valores $F$ calculados de las dos bases de datos son mayores que el valor $F$ crítico, lo que indica que se rechaza la hipótesis de estabilidad estructural y se acepta la del cambio estructural. Por lo cual, se determina que si se presentó un cambio estructural a partir de la reforma financiera de 2014 en el número de sucursales y en el número sociedades registradas de las SOCAPS de México. Ahora, en la Tabla 2, se presentan los resultados obtenidos de aplicar la prueba de Chow en los créditos vigentes totales de todas las SOCAPS de México, y a su vez divididos en créditos comerciales, al consumo y a la vivienda (el cambio estructural en estas cuatro bases de datos empezó en febrero de 2014).

Tabla. 2. Prueba de Chow (F) aplicado a los créditos vigentes totales de las SOCAPS de México y divididos en créditos comerciales, al consumo y a la vivienda.

\begin{tabular}{lccc}
\hline Variable & Valor F calculado & Valor F crítico & Se rechaza la hipótesis de estabilidad estructural \\
& & & \\
\hline Cartera de crédito vigente & 48.514 & 3.076574309 & Si se rechaza \\
Créditos comerciales & 10.058 & \\
Créditos al consumo & 67.088 & \\
Créditos a la vivienda & 55.449 & \\
\hline & Fuente: Elaboración propia.
\end{tabular}

Basándose en los resultados exhibidos en la Tabla 2, se puede determinar que en los créditos vigentes totales, en los créditos al consumo, en los créditos a la vivienda y en los créditos comerciales, sí existió un cambio estructural. Lo anterior es debido a que los valores $F$ calculados son mayores que el valor $F$ crítico, lo que señala que se rechaza la hipótesis de estabilidad estructural y se acepta la del cambio estructural. Por los resultados obtenidos y exhibidos en la Tabla 2, podemos establecer que la reforma financiera de 2014 provocó un cambio estructural en los créditos totales vigentes de las SOCAPS y que una vez divididos los créditos vigentes, se encuentra que la reforma financiera de 2014 también provocó un cambio estructural en los créditos al consumo, a la vivienda y los comerciales.

\subsection{Prueba de análisis estructural con variables dicótomas}

A continuación, se presentan los resultados de aplicar la prueba de análisis estructural con variables dicótomas a las seis bases de datos obtenidas de las SOCAPS, con el fin de sustentar y ampliar los resultados obtenidos en la prueba de Chow y determinar que sí existió un cambio estructural, este fue un cambio estructural en la pendiente o en el intercepto de la ordenada al origen. Los resultados obtenidos de aplicar el análisis estructural con variables dicótomas a las bases de datos que corresponde al número de sociedades registradas y al número de sucursales de las SOCAPS de México se muestran en la Tabla 3 (en estas bases de datos el cambio estructural comenzó en junio de 2014). 
Tabla. 3. Resultados de aplicar la prueba de análisis estructural con variables dicótomas al número de sociedades y sucursales de las SOCAPS de México.

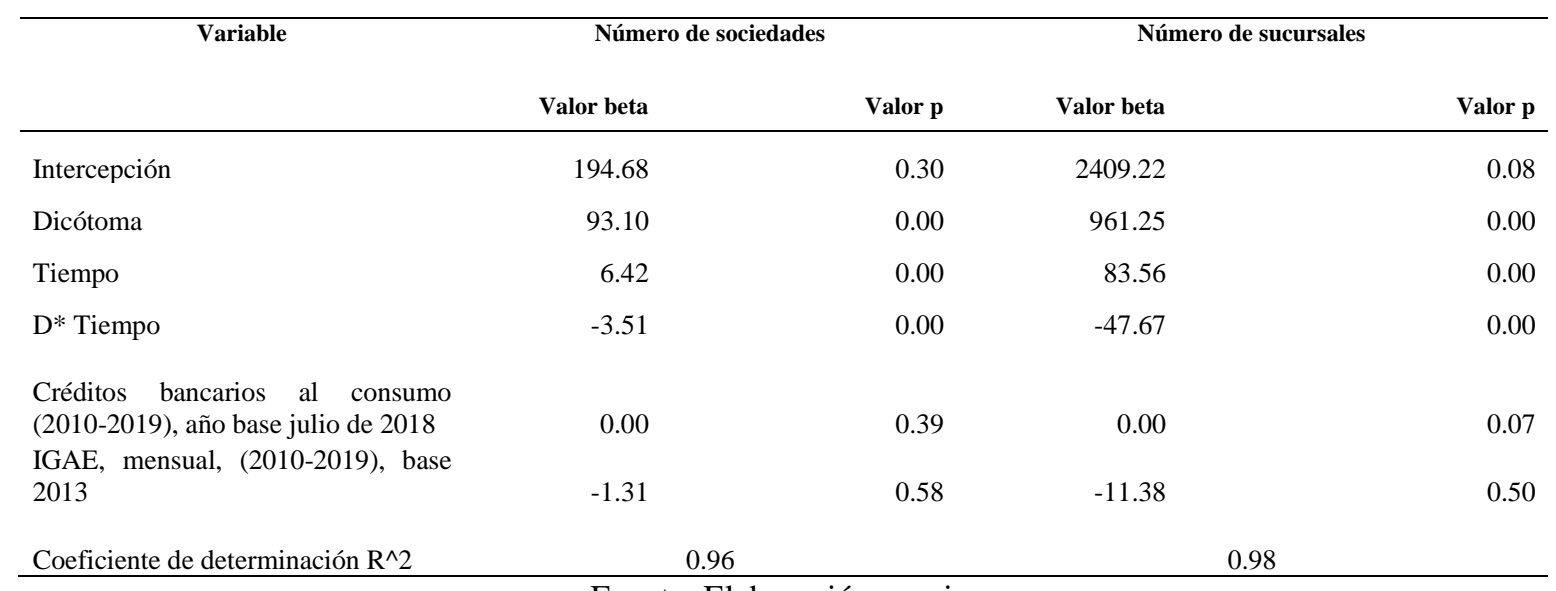

Fuente: Elaboración propia.

De acuerdo con los resultados mostrados en la Tabla 3 y basándose en el valor $R^{2}$ de los dos modelos, ambos son representativos ya que son superiores al .80. Ahora, analizando el valor $p$ del número de sociedades, los valores que tuvieron un valor $p$ menor a 0.05 fueron: la variable dicótoma; la variable de tiempo; y, la multiplicación entre el tiempo y la variable dicótoma. Las únicas variables que sus valores $p$ son mayores al 0.05 son el IGAE y los créditos bancarios al consumo. Basándose en los resultados de la Tabla 3, se puede establecer que en el número de sociedades registradas de las SOCAPS de México, sí se presentó un cambio estructural en el año 2014 y este provocó un cambio en la pendiente debido a que el valor $p$ de la pendiente diferencial (D*Tiempo) es menor a 0.05 ; y, en el intercepto de la ordenada al origen ya que el valor $p$ del intercepto diferencial (Dicótoma) también es menor a 0.05 .

Por otro lado, respecto al número de sucursales, los valores $p$ que son significativos al ser menores al 0.05 son: la variable dicótoma; el tiempo; y, la multiplicación entre el tiempo y la variable dicótoma. En cambio, los créditos bancarios al consumo y el IGAE sus valores $p$ son mayores al 0.05 , por lo cual no son significativos. Con base en los resultados mostrados en la Tabla 3, se puede determinar que si se presentó un cambio estructural en el año 2014 en el número de sucursales y este causó un cambio tanto en la pendiente como en la ordenada al origen, debido a que los valores $p$ del coeficiente de la pendiente diferencial (D*Tiempo) y del intercepto diferencial (Dicótoma), son menores a 0.05 .

Los resultados mostrados en la Tabla 3 sustentan a los obtenidos por la prueba de Chow, que la reforma financiera de 2014 realizada en México si causó un cambio estructural en el número de sociedades registradas y en el número de sucursales de las SOCAPS de México. Se debe resaltar que el análisis con variables dicótomas amplía los resultados obtenidos por los de Chow, ya que estos señalan que tanto en el número de sucursales como en el número de sociedades registradas, el cambio estructural fue tanto en la pendiente como en la ordenada al origen. Continuando con el análisis, en la Tabla 4 se presentan los resultados de aplicar la prueba de análisis estructural con variables dicótomas en los créditos vigentes de las SOCAPS y divididos en créditos al consumo, a la vivienda y comerciales (el cambio estructural en estas cuatro bases de datos empezó en febrero de 2014). 
Tabla. 4. Resultados de aplicar la prueba de análisis estructural con variables dicótomas a los créditos vigentes de las SOCAPS de México y divididos en créditos al consumo, a la vivienda y comerciales.

\begin{tabular}{|c|c|c|c|c|c|c|c|c|}
\hline & \multicolumn{2}{|c|}{ Créditos vigentes } & \multicolumn{2}{|c|}{ Créditos comerciales } & \multicolumn{2}{|c|}{ Créditos al consumo } & \multicolumn{2}{|c|}{ Créditos a la vivienda } \\
\hline & Valor beta & Valor $p$ & Valor beta & Valor $p$ & Valor beta & Valor p & Valor beta & Valor $p$ \\
\hline Intercepción & 107562975 & 0.000 & 93734098.72 & 0.000 & -2115139.817 & 0.930 & 15944016.1 & 0.000 \\
\hline Dicótoma & -9308794.319 & 0.000 & 2287920.993 & 0.078 & -10646222.42 & 0.000 & -950492.8884 & 0.000 \\
\hline Tiempo & 481852.3566 & 0.000 & 388132.2111 & 0.000 & -4254.658339 & 0.961 & 97974.8039 & 0.000 \\
\hline D*tiempo & 249739.2562 & 0.000 & -45908.18727 & 0.115 & 285282.831 & 0.000 & 10364.61249 & 0.009 \\
\hline $\begin{array}{l}\text { Créditos bancarios al } \\
\text { consumo (2010-2019), año } \\
\text { base julio de } 2018\end{array}$ & -34.76652217 & 0.002 & -17.04270763 & 0.025 & -8.669535123 & 0.481 & -9.054279421 & 0.000 \\
\hline $\begin{array}{l}\text { IGAE, mensual, (2010- } \\
\text { 2019), base } 2013\end{array}$ & -480928.6056 & 0.058 & -919904.503 & 0.000 & 537033.089 & 0.064 & -98057.19166 & 0.000 \\
\hline $\begin{array}{l}\text { Coeficiente de } \\
\text { determinación } \mathrm{R}^{\wedge} 2\end{array}$ & \multicolumn{2}{|c|}{0.982910803} & \multicolumn{2}{|c|}{0.842423385} & \multicolumn{2}{|c|}{0.956732917} & \multicolumn{2}{|c|}{0.981281693} \\
\hline
\end{tabular}

Fuente: Elaboración propia.

De acuerdo con los datos presentados en la Tabla 4, las cuatro regresiones son representativas porque tienen un valor $R^{2}$ superior a .8 lo cual las hace significativas. Respecto al análisis de los valores $p$, se puede encontrar que en los créditos vigentes de las SOCAPS, las variables que tienen un valor $p$ menor a 0.05 son todas con excepción del IGAE. En este sentido, los resultados indican que las reformas financieras de 2014 si causaron un cambio estructural tanto en la pendiente como en la ordenada al origen de los créditos vigentes dados por las SOCAPS, y esto es debido a que los valores $p$ del coeficiente de la pendiente diferencial (D*Tiempo) y del intercepto diferencial (Dicótoma) son menores a 0.05 lo que las hace estadísticamente significativas.

Por otro lado, en los créditos comerciales dados por las SOCAPS los valores $p$ significativos, es decir, que son menores a 0.05 son el IGAE, los créditos bancarios al consumo y el tiempo, mientras que las otras variables resultaron que no son significativos ya que tuvieron valores $p$ mayores a 0.05 . También debido a que las variables $\mathrm{D} *$ tiempo (coeficiente de la pendiente diferencial) y la dicótoma (intercepto diferencial), tienen valores $p$ mayores a 0.05 , se determina que no hay un cambio estructural ni en la pendiente ni en la ordenada al origen de los créditos comerciales concedidos por las SOCAPS de México.

En cambio, en los resultados obtenidos de aplicar la prueba de análisis estructural con variables dicótomas en los créditos al consumo dados por las SOCAPS de México, se halló que los valores $p$ que son significativos debido a que tuvieron valores menores a 0.05 son: la variable dicótoma y la multiplicación entre la variable dicótoma y el tiempo. En cambio, las variables tiempo, créditos bancarios al consumo y el IGAE no son significativos ya que sus valores $p$ son mayores a 0.05 . Los resultados obtenidos indican que si se presentó un cambio estructural en 2014 en los créditos al consumo otorgados por las SOCAPS derivado de las reformas financieras del mismo año, y, este fue tanto en la pendiente como en la ordenada al origen, ya que el valor $p$ del coeficiente de la pendiente diferencial (D*Tiempo) y del intercepto diferencial (Dicótoma) son menores a 0.05 , lo que las hace estadísticamente significativas.

Por último, en los créditos a la vivienda concedidos por las SOCAPS de México y mostrados en la Tabla 4 , se encontró que todas las variables son significativas ya que sus valores $p$ son menores a 0.05 . En este sentido, si hubo un cambio estructural en los créditos a la vivienda dados por las SOCAPS y este cambio fue tanto en la pendiente como en la ordenada al origen, debido a que el valor $p$ del intercepto diferencial (Dicótoma) y el valor $p$ de la pendiente diferencial (D*Tiempo) son menores a 0.05 .

Los resultados mostrados en la Tabla 4 concuerdan con los que se obtuvieron en la prueba de Chow, es decir, que las reformas financieras de 2014 si causaron un cambio estructural en los créditos vigentes otorgados por las SOCAPS, pero añadiendo que fue un cambio en la pendiente y en el intercepto de la ordenada al origen. Sin embargo, una vez que se dividen los créditos vigentes en créditos al consumo, a la vivienda y comerciales, se encontró que si hay un cambio estructural en los créditos al consumo y a la vivienda y este fue tanto en la pendiente como en la ordenada al origen; pero no en los créditos comerciales (ni en la pendiente ni en la ordenada al origen). Respecto a los créditos comerciales, se debe indicar que los resultados de la prueba de análisis estructural contradicen a los de Chow (en estos se determinaron que si hubo un cambio estructural), lo cual provoca que los resultados no sean concluyentes. No obstante, se debe 
resaltar que ambas pruebas se realizaron con un valor $\alpha$ del cinco por ciento, es decir, al $95 \%$ y que si este se ampliara al diez por ciento, si habría un cambio estructural en los créditos comerciales.

Aun así, los resultados obtenidos en esta sección respaldan y complementan a los conseguidos en la prueba de Chow. Esto se debe a que las dos pruebas de análisis estructural coinciden en sus resultados (exceptuando los créditos comerciales, bajo las condiciones ya explicadas) y que se pudo determinar que en todos los casos donde se presentaron cambios estructurales, estos fueron tanto en la pendiente como en la ordenada al origen.

\subsection{Discusión teórica de los resultados}

De acuerdo con los resultados obtenidos por medio de las pruebas de análisis estructural o Chow y con variables dicótomas, se determina que las reformas financieras de 2014 si tuvieron un efecto en las Sociedades Cooperativas de Ahorro y Préstamo (SOCAPS) de México. En este sentido, se pudo establecer que estas reformas financieras si tuvieron un efecto en el número de sucursales y en el número de sociedades registradas (las variables empleadas para describir el comportamiento de las SOCAPS). Asimismo, que las reformas también tuvieron un efecto que causó un cambio estructural en el total de créditos vigentes y que una vez divididos estos en créditos al consumo, a la vivienda y comerciales (variables proxis de la inclusión financiera), se halló que si se presentó un cambio estructural tanto en los créditos al consumo como a la vivienda, no obstante los resultados no fueron concluyentes para los créditos comerciales (en Chow la evidencia estadística señala que si hubo un cambio estructural, pero en la prueba con variables dicótomas no se halló evidencia).

Estos resultados son de variables proxis de la inclusión financiera y describen el comportamiento de las SOCAPS de México. En este sentido, en las bases de datos del número de sucursales y de sociedades registradas de las SOCAPS, que se presentaron en la Figura 1, se puede observar que tienen un crecimiento positivo (que es sustentado por las líneas de tendencia que se ven en la misma figura). También, basándose en la prueba de Chow, se confirmó que si hubo un cambio estructural en el número de sucursales y de sociedades registradas de las SOCAPS, mientras que con la prueba de análisis estructural, se determinó que tanto en el número de sucursales como en el número de sociedades registradas, el cambio fue tanto en la pendiente como en la ordenada al origen.

Ahora, apoyándose en lo anterior, es decir, una vez que se ha determinado que si hubo un cambio estructural y basándose en la Figura 1 donde se observa que el número de sucursales y de sociedades registradas han crecido. Se puede establecer que las reformas financieras si tuvieron un impacto que causó un cambio estructural y que este fue positivo, lo cual, provocó que las SOCAPS de México crecieran. Dicho de otra manera, las reformas financieras de 2014 tuvieron una influencia positiva en el número de sucursales y en el número de sociedades; este cambio tuvo un impacto positivo en la pendiente y en el intercepto de la ordenada al origen, lo que indica que estas reformas causaron un incremento en el número de sucursales y en el número de sociedades registradas, $\mathrm{y}$, además tuvieron un impacto positivo en la tendencia del crecimiento de ambas variables.

Respecto a la base de datos de datos de los créditos (variables proxis de la inclusión financiera), se encontraron resultados similares a los de las variables de crecimiento. En este sentido, en la Figura 2 se puede observar que en las cuatro bases de datos (que corresponden a los créditos vigentes y estos divididos en créditos al consumo, a la vivienda y comerciales) la pendiente de su línea de tendencia es positiva, lo que señala que han crecido. Por otro lado, en las pruebas de Chow y en la de análisis estructural con variables dicótomas, se encontró que si se presentó un cambio estructural en el año 2014, lo que sustenta que las reformas financieras de 2014 tuvieron un impacto. La única excepción fueron los créditos comerciales, en los cuales los resultados no fueron concluyentes.

De acuerdo con lo anterior, se puede establecer que la reforma financiera de 2014 si causó un cambio estructural en los créditos vigentes de las SOCAPS y que este fue positivo. Asimismo, la evidencia estadística señala que el cambio fue tanto en la pendiente como en el intercepto, o sea, que la reforma financiera causó un aumento en el número de créditos vigentes de las SOCAPS, pero, además un cambio positivo en la tendencia del crecimiento de estos créditos.

Por otro lado, analizando por separado los créditos vigentes, se halló que en el caso de los créditos al consumo y a la vivienda, las reformas también causaron un cambio estructural y que este fue positivo. En estas dos sub divisiones el cambio fue tanto en la pendiente como en la ordenada al origen, es decir, el cambio estructural derivado de la reforma financiera de 2014, provocó un incremento en los créditos otorgados a la vivienda y al consumo, pero, también en la tendencia del crecimiento de estas (que fue positivo). Respecto a los créditos comerciales, los resultaron no fueron concluyentes. 
Es importante recordar que el objetivo de la reforma financiera de 2014 fue ampliar y facilitar el acceso al crédito, y específicamente a los microcréditos, para este último, fue que se reformó la Ley Para Regular las Actividades de las Sociedades Cooperativas de Ahorro y Préstamo (LPRSCAP) (Rodríguez y Dorantes, 2016; LRASCAP, 2019). En este sentido, se puede determinar que a partir del análisis realizado a las seis bases de datos de las SOCAPS la reforma financiera de 2014 si tuvo un impacto en estas (es decir, hubo un cambio estructural) y este fue positivo.

Por un lado, las pruebas aplicadas a las variables usadas en esta investigación, permiten afirmar que a partir del año 2014 (donde se presentó el cambio estructural) las SOCAPS crecieron, ya que se incrementó el número de sucursales lo que les permite atender a un mayor número de personas, también aumentaron el número de sociedades registradas, lo que ayuda a poder llegar a un mayor número de individuos. Asimismo, la tendencia del crecimiento de las SOCAPS también fue afectada de manera positiva, ósea, la tendencia de su crecimiento fue mayor.

Por otro lado, el análisis de las variables referentes a los créditos otorgados por las SOCAPS permite establecer que la reforma financiera de 2014 también causó un cambio estructural que contribuyó a que estos créditos se incrementaron y que la tendencia de su crecimiento fuera mayor. Lo cual era un objetivo de la reforma financiera. Es importante resaltar que, si bien la evidencia presentada en esta investigación indica que los créditos emitidos por las SOCAPS en general fueron afectados positivamente por las reformas, este efecto fue solo en los créditos destinados a la vivienda y al consumo, pero, en los créditos comerciales la evidencia no fue concluyente.

Lo anterior lleva a establecer que, dada la evidencia obtenida del análisis de las seis bases de datos de las SOCAPS, la reforma financiera de 2014 si tuvo un impacto positivo en estas y que alcanzó los objetivos por los cuales se implementó. Asimismo, que las SOCAPS si han crecido a partir de la reforma financiera de 2014, lo cual les permitió ampliar la cantidad de servicios ofrecidos, lo que ayudó a que aumentaran los créditos concedidos por estos y, por lo tanto, impactara positivamente a la inclusión financiera. El análisis de las variables proxis de la inclusión financiera, permitieron establecer que el crecimiento de las SOCAPS también contribuyó de forma positiva a la inclusión financiera.

\section{Conclusiones}

El objetivo de la investigación fue determinar si las reformas de 2014 a la Ley para regular las actividades de las sociedades cooperativas de ahorro y préstamo, tuvieron un impacto en el crecimiento de las Sociedades Cooperativas de Ahorro y Préstamo de México que fomentaran la inclusión financiera. Por ello, se analizaron seis bases de datos de las Sociedades Cooperativas de Ahorro y Préstamo de México (SOCAPS) que permitieran alcanzar el objetivo planteado. Por un lado, dos de ellas referentes a describir el comportamiento de las SOCAPS y que son: el número de sucursales y el número de sociedades registradas. Por el otro, cuatro de ellas como variables proxis de la inclusión financiera y que son el total de créditos vigentes de las SOCAPS y a la vez; estos divididos en créditos al consumo, a la vivienda y comerciales (estas están expresadas en unidades monetarias).

Estas seis bases de datos se analizaron con el fin de establecer si hubo o no un cambio estructural por medio de la prueba de Chow y la prueba de análisis estructural con variables dicótomas. Esta última permitió determinar en donde fue el cambio estructural. Los resultados obtenidos de las dos pruebas permiten establecer que si hubo un cambio estructural en el año 2014 en cinco de las seis bases de datos analizadas en la investigación, y, que en estas cinco el cambio estructural fue en la pendiente y en el intercepto al origen (en una de ellas, la evidencia estadística no fue concluyente).

La evidencia permite concluir que si hubo un cambio estructural derivado de la reforma financiera de 2014 y que fue positivo. Esto permite establecer que las SOCAPS crecieron, es decir, el número de sucursales y sociedades registradas aumentaron y que su tendencia de crecimiento fue mayor. Por otro lado, respecto a los créditos vigentes de las SOCAPS se concluye que también se incrementaron y que la tendencia de su crecimiento fue superior a la mostrada previa a la reforma financiera de 2014. Asimismo, que una vez divididos los créditos vigentes en: créditos al consumo, a la vivienda y comerciales. Se concluyó que estos también aumentaron y que su tendencia de crecimiento fue mayor, la única excepción fueron los créditos comerciales en los cuales la evidencia no permitió llegar a alguna conclusión. Estas bases de datos se consideran variables proxis de la inclusión financiera, por lo cual, se pudo establecer que si hubo un impacto positivo en la inclusión financiera derivado del crecimiento de las SOCAPS.

Además, se puede concluir que los objetivos por los cuales se implementó la reforma financiera de 2014 se alcanzaron. Debido a lo anterior, se puede establecer que se alcanzó el objetivo principal de la investigación. Los resultados y las conclusiones de esta investigación son relevantes para las autoridades 
encargadas de elaborar las leyes que regulan el sector de las sociedades cooperativas, los investigadores afines al tema y los administradores de las sociedades cooperativas. Como futuras líneas de investigación, se sugiere analizar la distinción entre los tres créditos, para determinar cuál de ellos es mejor para el desarrollo económico de la región donde opera la SOCAP.

\section{Referencias bibliográficas}

Banco de México (BANXICO) Financiamiento e información financiera de intermediarios financieros, Banca Comercial, Cartera vigente otorgada al sector privado no bancario (metodología 2018), Banca comercial, Cartera de crédito vigente total al sector privado $1 /$, Saldos nominales en millones de pesos, B. Cartera vigente total por destino del crédito, B.1 Consumo. México. Fecha de consulta: 6 de junio de 2019. Extraído de http://www.banxico.org.mx/SieInternet/consultarDirectorioInternetAction.do?sector=19\&idCuadro=CF835\&accion $=$ consultarCuadro\&locale $=$ es.

Cabrera, A. (2015) Trascendencia de las cooperativas en México: Análisis documental del impacto socioeconómico en comunidades rurales. Strategy, Technology \& Society, pp. 104-120.

Calvo Bernardino, A. y Paúl Gutiérrez, J. (2010) Las cajas de ahorro y las cooperativas de crédito ante la crisis: evolución en su presencia territorial y en su operativa. REVESCO, Revista de Estudios Cooperativos, № 100, pp. 68-100.

Cárdenas Félix, C. J., Hirsch, J. y Lara Gómez, G. (2015) Estructuras contractuales en el sector de las finanzas populares en el municipio de Querétaro en México. CIRIEC-España, Revista de Economía Pública, Social y Cooperativa, $\mathrm{N}^{\circ}$ 84, pp. 133-162. ISSN: 0213-8093.

Cobián, A., Ortiz, M., L., Joya, R., y Núñez, A. (2016) Indicadores de contribución al desarrollo sostenible de una caja de ahorro y préstamo. Retos de la Dirección, Vol. 10, No 2, pp. 90-107. ISSN 2306-9155.

Comisión Nacional Bancaria y de Valores (CNBV) Portafolio de Inversión, Boletines Estadísticos de las Sociedades Cooperativas de Ahorro y Préstamo, estados financieros condensados. México, varios años. Fecha de consulta: 4 de agosto $\quad 2019.2$ Extraído de https://portafolioinfo.cnbv.gob.mx/Paginas/Contenidos.aspx?ID=29\&Titulo=Sociedades $\% 20$ Cooperativas $\% 20 \mathrm{de} \% 2$ 0Ahorro\%20y\%20Pr\%C3\%A9stamo.

Comisión Nacional Bancaria y de Valores (CNBV) Información de la situación financiera: Sociedades Cooperativas de Ahorro y Préstamo, Estados financieros condensados. México. Fecha de consulta: junio de 2019. Extraído de https://portafolioinfo.cnbv.gob.mx/Paginas/PIExcel/sc infosituacion.aspx.

Figueroa, L. y Magaña, D. (2018) Las sociedades cooperativas de ahorro y préstamo en México la teoría de la empresa alternativa, social y pluralista (stakeholder). Alegatos, $\mathrm{N}^{\circ} 99$, pp. 269-286.

Gujarati, D., y Porter, D. (2010) Econometría. 5ta. edición. México, DF: Mc Graw Hill.

Instituto Nacional de Estadística y Geografía (INEGI) Índice Nacional de Precios al Consumidor (INPC). México. Fecha de consulta: julio de 2019. Extraído de https://www.inegi.org.mx/temas/inpc/.

Instituto Nacional de Estadística y Geografía (INEGI) Índice Global de la Actividad Económica (IGAE). México. Fecha de consulta: julio de 2019. Extraído de https://www.inegi.org.mx/programas/igae/2013/default.html\#Tabulados.

Lara Gómez, G. (2010) De cajas populares a cooperativas de ahorro y préstamo. Algunas evidencias. Revista Estudios Agrarios, Vol. 16, No 45, pp. 121.

Lara Gómez, G. (2012) Cambio Organizacional en una Federación de Ahorro y Crédito Popular. México: Plaza y Valdés.

Lara Gómez, G. (2018 a) Los incentivos tributarios para las cooperativas financieras de Colombia, Costa Rica y México. REVESCO. Revista de Estudios Cooperativos, Primer Cuatrimestre, $\mathrm{N}^{\circ}$ 127, pp. 159-180. http://dx.doi.org/10.5209/REVE.59772.

Lara Gómez, G. (2018 b) Los gastos tributarios y las exenciones para las cooperativas en Costa Rica y México. Quaestio Iuris, volumen 11, No 04, pp. 2989-3012. DOI: https://doi.org/10.12957/rqi.2018.37281.

Lara Gómez, G., y Pérez Sosa, F. A. (2015) Determinantes del isomorfismo institucional de las sociedades cooperativas de ahorro y préstamo en México. REVESCO. Revista de Estudios Cooperativos, Tercer Cuatrimestre, № 119 , pp. 77-106. DOI: https://doi.org/10.5209/rev_REVE.2015.n119.49068.

Lara Gómez, G., Pérez Sosa, F. A., y Hurtado Maldonado, J. (2017) Finanzas Populares en México. Primera edición. México: Fontamara.

Ley de ahorro y crédito popular (2019) Diario Oficial de la Federación, H. Congreso de la Unión. Recuperado de https://www.cnbv.gob.mx/Normatividad/Ley\%20de\%20Ahorro\%20y\%20Cr\%C3\%A9dito\%20Popular.pdf.

Ley para regular las actividades de las sociedades cooperativas de ahorro y préstamo (2019) Diario Oficial de la Federación, H. Congreso de la Unión. Recuperado de http://www.diputados.gob.mx/LeyesBiblio/pdf/LRASCAP_280414.pdf.

Martínez-Carrasco, F., Muñoz, A., Eid, M., y Colino, J. (2016) Inclusión financiera en el ámbito rural mediante cajas de ahorro. Estudio de una experiencia en México. Perfiles Latinoamericanos, Vol. 24, № 48, pp. 185-211.

Masís Solano, P., Gómez Pescador, I. y Arzadun, P. (2016) Iniciativas sociales, económicas y ambientales: su impacto en la opinión de la base asociativa de una Cooperativa de Ahorro y Crédito de Costa Rica. CIRIEC-España, Revista de Economía Pública, Social y Cooperativa, No 86, pp. 100-122. ISSN: 0213-8093. 
Medina, A., y Flores, U. (2015) Estudio jurídico y fiscal de las sociedades cooperativas como empresas de carácter social en la región mixteca Oaxaca, México y su situación actual. Boletín de la Asociación Internacional de Derecho Cooperativo, No 49, pp. 251-228. ISSN: 1134-993X o ISSN-e: 2386-489.

Ollivier, J. Ó. y Thompson, P. I. (2017) Guía para elaborar trabajos de investigación. Chihuahua, México: Universidad Autónoma de Chihuahua.

Rodríguez, A., y Dorantes, P. M. (2016) La reciente reforma financiera en México: Transformaciones y perspectivas. ECONOMÍAunam, Vol. 13, No 37, pp. 89-106. ISSN:1665-952x.

Torres Grimaldo, J. A., y Cano Morales, A. M. (2019) Importancia del Gobierno Corporativo en las sociedades financieras populares en México. Cuadernos de contabilidad, Vol. 20, $\mathrm{N}^{\circ} 49 . \quad \mathrm{DOI}$ : https://doi.org/10.11144/Javeriana.cc20-49.igcs.

Vargas Vasserot, C. (2015) Las cooperativas de crédito y su posición dentro del modelo cooperativo. Integración frente a diferenciación en el marco de la reforma del sistema financiero. REVESCO. Revista de Estudios Cooperativos, Primer Cuatrimestre, No 117, pp. 50-76. DOI: https://doi.org/10.5209/rev_REVE.2015.v117.48145. 